\title{
Radiation Doses and Cancer Risk from CT Pulmonary Angiography examinations
}

\author{
H H Harun ${ }^{\text {a }}$, M K A Karim ${ }^{\mathrm{a}, \mathrm{b}}$ *, Z Abbas a ${ }^{\mathrm{a}}$, A Sabarudin ${ }^{\mathrm{c}}$, S C Muniandy ${ }^{\mathrm{d}}$ and K H Ng ${ }^{\text {e }}$ \\ a \\ b \\ Selangor, Malaysia \\ c Department of Diagnostic \& Applied Health Sciences, Faculty of Health Sciences, Universiti Kebangsaan \\ Malaysia, 56000, Kuala Lumpur, Malaysia \\ d Department of Radiology, Hospital Kuala Lumpur, Jalan Pahang, 50586 Kuala Lumpur, Malaysia \\ e Department of Biomedical Imaging, Faculty of Medicines, Universiti Malaya, 50603 Kuala Lumpur, \\ Malaysia \\ Department of Physics, Faculty of Science, Universiti Putra Malaysia, 43400 Serdang, Selangor, Malaysia \\ Center for Diagnostic Nuclear Imaging, Faculty of Medicine, Universiti Putra Malaysia, 43400 Serdang,
}

*Corresponding author: khalis.karim@gmail.com

\begin{abstract}
The present study aims to investigate radiation doses from Computed Tomography Pulmonary Angiography (CTPA) examinations based on the patient's size and to estimate the probability of cancer risk induced from the examination. Data from 100 patients who had undergone CTPA examinations, such as scanning acquisition parameters, patient demography, as well as radiation dose exposure, were collected and analysed. All subjects which aged above 18 y/o were scanned using a Philips Brilliance 128 multidetector CT (MDCT) scanner. The mean dose value for Volume Computed Tomography Dose Index $\left(\mathrm{CTDI}_{\mathrm{vol}}\right)$, Dose-Length Product (DLP) and effective dose (E) were 11.06 $\pm 7.17 \mathrm{mGy}, 400.38 \pm 259.10$ mGy.cm and $8.68 \pm 5.47 \mathrm{mSv}$ respectively. In addition, with respective of patient's effective diameter, the mean SSDE value for Group 1, Group 2 and Group 3 were $9.93 \pm 3.89,13.70 \pm 9.04$ and $22.29 \pm 7.35$, respectively. Cancer risk per million procedure was calculated based on te recommendation by the International Commission on Radiological Protection Publication 103 report. The organ dose and cancer risk attained for breast, lung and liver were $17.05 \pm 10.40 \mathrm{mGy}$ (194 per one million procedure), $17.55 \pm$ $10.86 \mathrm{mGy}$ (192 per one million procedure) and $15.04 \pm 9.75 \mathrm{mGy}$ ( 53 per one million procedure), respectively. In conclusion, $\mathrm{CTDI}_{\mathrm{vol}}$ underestimated, and SSDE was more accurate in describing the radiation dose. Lung and breast with larger lung effective diameter received the highest dose exposure which increase the probability of the cancer risk. Therefore, it is important to apply optimised protocols in order to reduce patient's exposure during CTPA examination.
\end{abstract}

Keywords: Computed Tomography; radiation dose; cancer risk; CTPA; lung effective diameter

\section{Introduction}

Computed tomography $(\mathrm{CT})$ is a vital tool for screening, diagnosing and managing patient care. It has become the most preferred imaging method, and its use is increasing significantly - most excellent tools in diagnostic imaging, where the number of examinations using this technique is considerably growing. However, in 2001, the International Commission on Radiological Protection (ICRP) has expressed concern over the high use of CT in medicine after it reported that the radiation exposure was more significant compared to other imaging modalities [1]. CT Pulmonary Angiography (CTPA) allows the visualization of pulmonary arteries to facilitate diagnosis and treatment of pulmonary embolism (PE). Despite having high 
accuracy, CTPA has been observed to expose patients, and medical personnel to ionizing radiation doses of more than $10 \mathrm{mSv}$, which epidemiological data suggest may increase the risk of cancer $[2,3]$.

The radiation dose produced by CT scanners can calculate as the calculation of volumetric Computed Tomography Dose Index $\left(\mathrm{CTDI}_{\mathrm{vol}}\right)$. It based on CT parameters used during the scanning of a standard phantom as a surrogate patient [4]. However, $\mathrm{CTDI}_{\mathrm{vol}}$ estimates based on a phantom have many potential errors as they do not consider the size of the body, which varies between patients, especially children. To overcome this, the size-specific dose estimates (SSDEs), which incorporates individual patient factors into the $\mathrm{CTDI}_{\mathrm{vol}}$ calculation, was introduced by the American Physicians Association (AAPM) in 2011. Instead of basing dose calculation solely on a phantom, SSDE requires the input of individual patient size in the CT scanner [3]. AAPM also published a specific size conversion factor to accurately estimate the absorption properties of various body sizes and their doses. This conversion factor is multiplied with $\mathrm{CTDI}_{\mathrm{vol}}$ to obtain the SSDE [4].

Previous studies were published the estimation of SSDE by the method introduced by AAPM. It found that the ratio of SSDE with $\mathrm{CTDI}_{\text {vol }}$ is inversely proportional to the size of the patient. When the size of the patient increases, the ratio becomes decreases [5]. Most of the CT-system is used $32 \mathrm{~cm}$ body phantom calculation for determining the $\mathrm{CTDI}_{\mathrm{vol}}$ contribute for this finding. For CTPA, several studies that focused on CT dose and its optimization techniques [2,7-9]. Surprisingly, there is no known comprehensively studied describing SSDE in different size of the patient in CTPA examination. The most promising assessment is estimating the organ dose and cancer risk according to the patient's body habitus. Both assessments varied in different conditions, dependency on age, sex and population studied [10]. The limitation of radiation dose estimation may overcome by these assessments tailored to an individual patient exposure rather than a general population.

The $7^{\text {th }}$ report on Biological Effects of Ionizing Radiation (BEIR VII Phase 2) by the United States National Academy of Sciences popularly cited in estimating the cancer risk-based in radiological scans. Most studies reported that detrimental effects of radiation exposure are higher in susceptible patient populations, especially young women and children [11] As such, it is crucial to calculate an accurate organequivalent dose before obtaining an estimation of cancer risk. So far, no research has been found that evaluated the organ dose and cancer risk in CTPA examination based on previous study elsewhere $[2,8,9,12]$. This study aims to investigate the usefulness and effect of SSDE and patient size in evaluating radiation dose in CTPA examination and to estimate the cancer risk for each scan and organ.

\section{Materials and methods}

The research protocol was approved by the ethics committee of the Ministry of Health (Malaysia) which waived patient consent form for the retrospective analysis with an approval ID: NMRR-18-3088-44138. The records of 100 adults who underwent CTPA were retrieved from a Hospital Kuala Lumpur, Malaysia. The subjects were above age 18 and they experienced the procedure between September 2018 to February 2019. All subjects were scanned using a Philips Brilliance 128 multi-detector CT (MDCT) scanner, and the images were reconstructed using the DICOM software. 
Scanning acquisition parameters and data such as subjects' gender, antero-posterior (AP) and lateral (LAT) body lengths, tube voltage $(\mathrm{kV})$, tube current $(\mathrm{mA})$, rotation time, pitch factor, $\mathrm{CTDI}_{\mathrm{vol}}$ and doselength-product (DLP) were recorded from scanner console. The AP and LAT lengths of each subjects' image were measured using digital calipers on the scanner console, at the mid-slice location of the transverse CT images. Only the scan data of pulmonary embolism scan were included, while cases with incomplete details and modified protocols were excluded.

CTPA was performed using 40 to $70 \mathrm{ml}$ of iodinated contrast medium, followed by $50 \mathrm{ml}$ saline chaser which were intravenously injected into the subjects at a flow rate of $5 \mathrm{ml} / \mathrm{s}$. The bolus tracker technique was performed by placing the region of interest (ROI) on the main pulmonary trunk. The scan was started after 3 to 14 seconds with a threshold of $70 \mathrm{HU}$. The z-dom modulation was activated before each scan. Images were reconstructed with $1 \mathrm{~mm}$ slice thickness and 512 x 512 matrix size. To enhance the CT images, the iDose $^{4}$ level 4 iterative reconstructive technique was used for post-processing of images. The radiographers performing the scan were well experienced in this protocol at least three years.

$\mathrm{CTDI}_{\mathrm{vol}}$, AP and LAT lengths obtained from the scanner console were used to calculate SSDE based on the American Association of Physicists in Medicine (AAPM) Report 204. For comparison purposes, the SSDE were also estimated using CT-EXPO Ver 2.3.1 (SASCRAD, Bucholz, der Norheide, Germany) and Monte Carlo simulation based on data from a systematic survey. Derivation of SSDE, according to AAPM, report 204 began with the calculation of the effective diameter of the subjects' body, as stated in Equation 1.

$$
\text { Effective Diameter }(\mathrm{cm})=\sqrt{A P \times L A T}
$$

The effective diameters (body size) were normalized to the tabulated body size-dependent conversion factor (f-size) stated in the AAPM report 204. SSDE was calculated by multiplying the normalized f-size with the $\mathrm{CTDI}_{\mathrm{vol}}$ displayed on the scanner console as in equation 2.

$$
\text { SSDE }=\text { normalised } f-\text { size } x \text { CTDIvol }
$$

The $\mathrm{E}$ and organ dose are standard health risk indicators for organ and tissues exposed to ionizing radiation. The equation of $\mathrm{E}$ is as follow:

$$
E \approx k \times D L P
$$

Where the conversion factor of a representative thorax region $(\mathrm{k})$ obtained from the International Commission on Radiological Protection (ICRP) Publication 103 (2007) (ICRP 2007) are multiplied with DLP. The E and organ dose were estimated only by CT-EXPO software in this study.

The cancer risk for subjects' breast, lung and liver exposed to the CPTA primary beam was estimated using equation 4:

$$
R=\sum r_{T} \cdot H_{T}
$$


where $r_{T}$ is the nominal risk factor attained from the International Commission on Radiological Protection (ICRP) Publication 103 (ICRP 2007) and the $H_{T}$ is the organ-specific equivalent dose obtained by CTEXPO in breast, lung and liver respectively. The cancer risk $(\mathrm{R})$ per procedure was estimated by multiplying the organ dose with the risk factor $\left(r_{T}\right)$. Then the sum the cancer risk $(\mathrm{R})$ for each organ was estimated by equation 4.

Descriptive statistics are reported in this study as fraction and means with standard deviations. All data were entered SPSS V17.0 (SPSS, version 17.0 for Windows, Chicago, Illinois, USA) for statistical analysis. A p-value of $<0.05$ was considered to indicate statistically significant differences. Since the data was normal the independent t-test and one-way ANOVA test used in this study. The value involved from the protocol were presented in the tables and boxplots.

\section{Results}

Table 1 shows the mean baseline characteristics of study subjects compromising 42 men and 58 women. The ranged age for men was $23-71$, whereas it was $18-77$ for women. The effective diameters ranged from 20.14 to $37.48 \mathrm{~cm}$ in males and $19.71-32.25$ in females. Table 2 states the scanning parameters of this study. The tube voltage was set at 100 or $120 \mathrm{kV}$ depending on the subject's habitus. The tube current and scan range were adjusted depending on body size. The calculation of CTDI $_{\text {vol }}$, SSDE, DLP, E and the ratio of SSDE to CTDI ${ }_{\text {vol }}$ were grouped according to effective diameters and in total, as stated in Table 3

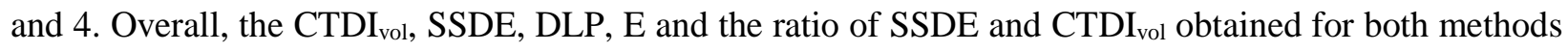
was $11.06 \pm 7.1,14.62 \pm 8.41$ vs $15.37 \pm 9.67,400.38 \pm 259.10,8.68 \pm 5.47$ and 1.41 vs 1.32 respectively. Surprisingly all SSDE values were higher than CTDI $_{\mathrm{vol}}$ for each patients' effective diameters group. The details of the observation as shown in Table 3, Table 4 and Figure 1.

Table 5 is a complication of scan parameters and dose results of other CTPA studies that used CTScanners by various manufacturers. All the studies produced different SSDE and CTDI ${ }_{\mathrm{vol}}$, DLP and E results because of the different pitch, tube current and beam collimation employed in their scans. The results of this study were similar to Sabel et al. (2016), but we measured the highest value of CTDI $_{\mathrm{vol}}$ compared with other studies. The SSDE value reported by Sabel et al. (2016) was the highest, around one-third more than this study [9]. It was likely because of our subjects' size, which was mostly larger compared with this study. The highest values of the DLP and E in this study and the lowest is a study done by Laqmani et al. (2016).

Table 6 states the individual and total organ dose for breast (females only), lung and liver. Table 7 states the cancer risk in a million procedures. The breast seemed to receive the highest organ dose in total, hence resulting in the cancer risk per million procedures. However, in the largest effective diameter, the lung had slightly higher dose exposure with similarly high cancer risk than the breast. The liver had the least dose exposure even though the values increased with effective diameter. However, its risk factor was extremely low- more than three times lower compared to the breast and lung. This breakdown of results between gender is also provided. Table 8 shows that the E, organ doses and cancer risk were all higher in females than males, but only the $\mathrm{E}$ was significant $(\mathrm{P}=0.04)$. Figure 1 shows the mean radiation doses and cancer risk. 


\section{Discussion}

The patient size varied along the Z-axis of the scan due to changes in the thickness and composition of the subjects' habitus. As expected, the variation of the subjects' effective diameter was contributed to the SSDE in line with a previous study [9]. Hence, the CTDI $_{\mathrm{vol}}$ calculated from the console and CT-EXPO software was observed to be undervalued compared to SSDE, especially in small-sized subjects. Overall, the reference phantom based $\mathrm{CTDI}_{\mathrm{vol}}$ values underestimated the radiation dose received by the patient as comparison by SSDE approach. The small variations in SSDE to CTDI ${ }_{\mathrm{vol}}$ ratio generated by CT-EXPO method in different subject sizes group was expected since the software's calculations were based on mathematical phantom [10]. However, the ratio generated by the AAPM report method was wider in a small-sized group compare to bigger-sized as the $f$-size increased in decreasing subjects' body size. A previous study had observed that when the automated exposure control (AEC) system was deployed, both $\mathrm{CTDI}_{\mathrm{vol}}$ and SSDE values were higher in large-sized subjects than those who were standard-sized. When the AEC system was turned off, there seems to be no significant difference between the radiation doses in different subject sizes [13]. This observation was aligned with this study where the AEC was deployed.

The variation in $\mathrm{CTDI}_{\mathrm{vol}}$ and SSDE were highly dependent on the scanning parameters. The use of low tube voltage had been reported as the most effective way to reduce the radiation dose exposure in CT examination especially CTPA $[12,14,15]$. A previous study had demonstrated that by lowering the tube voltage to $80 \mathrm{kV}$, it could reduce the $\mathrm{CTDI}_{\mathrm{vol}}$ to about $70 \%$ in contrast to this study [8]. However, the low tube had to be applied cautiously without affecting image quality. Another study reported that reducing the tube voltage in CT examination involve contrast media could maximize the photoelectric effect, as the applied voltage was closer to the K-edge of iodine $(33.2 \mathrm{keV})$. It could enhance the performance of image quality as well as to reduce the radiation dose [7]. The tube current indicated in Table 4 showed a similar trend in various studies. Most studies applied the AEC system to modulate the tube current and reduce unnecessary radiation exposure to their subjects [16-20]. However, the variation in pitch factor and beam collimation showed different approaches taken by various institutions in utilizing their CT-Scanner.

Theoretically, the scanning is controlled by increasing or decreasing the pitch factor, where the radiation dose received by patients would be reduced by increasing the pitch factor, but at the expense of image quality [21]. Modern scanners were developed to cover a wider beam collimation range per rotation of the CT X-ray tube. It would reduce the scanning time and radiation dose received by the patient [22]. The DLP value and effective diameter were dependent on the scan length and range, as stated in other studies $[2,7,9,23]$. However, there was limited information on the scan parameters. As the CTDI ${ }_{\text {vol }}$ and SSDE values in this study were high, the DLP and E could be expected to follow suit, as in Table 4. The dose calculation was highly dependent on the scan length and patient habitus, especially for DLP [24,25]. A previous study which also calculated the radiation dose exposure using AAPM Report 204 and CT-EXPO software, reported that the two methods had a deviation of around five per cent, which was similarly observed in this study [26].

It is clearly shown that the breast and lungs received the highest radiation dose exposure as these organs cover all in the primary beam. Both also had an equally high risk of developing cancer. On the other hand, the liver attained the lowest values in organ dose and cancer risk. It was mainly because, in a CTPA procedure, the liver would only be partially scanned as it was not entirely within the region of interest. This 
observation was in line with the BEIR VII report, which stated the dose exposure and cancer risk were dependent on the location of the organ from the primary beam, as well as their sensitivity to radiation. The higher tube current required to scan subjects with increasing body effective diameter was the main factor that caused the significant differences in organ doses and their cancer risk, as observed in Table 5 and 6. The AEC system automatically modulated the tube current according to patient size and habitus [27,28]. Thus, subjects with large body sizes were at higher risk when undergoing CTPA.

Unfortunately, the risk estimation in this study was not comparable with other studies, which were presented with various methodologies and different cases [29-31]. Although not significant, the result of this study supported previous research that found a higher cancer risk in females. The overall lethality risk for females was approximately $35 \%$ higher in comparison with males, as illustrated in Table 7 [10]. There were some limitations to this study. Firstly, the subjects in this study not involved with paediatrics and adolescents hence further investigation needed to evaluate the radiation dose and selected organ risk between these group of ages. Secondly, the SSDE values derived using CT-EXPO software was not normalized to the effective diameters of each patient. Thus, the values were not accurately estimated since the CT-EXPO software only utilized a fixed size mathematical phantom. However, this study overcomes this limitation with another evaluation by AAPM report 204 methods. Lastly, no alternative dose descriptor software beside CT-EXPO used in this study to calculate the e, organ dose and cancer risk. No comprehensive comparison of the value of these variables had been done in this study.

\section{Conclusions}

In conclusion, radiation dose exposure depends on various factors and would dramatically increase with the subjects' effective diameter. It is observed that radiation dose and cancer risk were significantly different between subjects' effective diameters, and E value has different between gender. Further study could focus on another group ages of subjects scan and additional dose descriptor software to compare the results with CT dosimetry calculator.

\section{References}

[1] Foley SJ, McEntee MF, Rainford LA. Establishment of CT diagnostic reference levels in Ireland. Br J Radiol 2012;85:1390-7.

[2] Sauter A, Koehler T, Brendel B, Aichele J, Neumann J, Noël PB, et al. CT pulmonary angiography: dose reduction via a next generation iterative reconstruction algorithm. Acta Radiol 2018;0:1-10.

[3] Halid B, Karim MKA, Sabarudin A, Bakar KA, Shariff ND. Assessment of lifetime attributable risk of stomach and colon cancer during abdominal CT examinations based on monte carlo simulation. IFMBE Proc., vol. 63, 2018, p. 455-9.

[4] Daudelin A, Medich D, Andrabi SY, Martel C. Comparison of methods to estimate waterequivalent diameter for calculation of patient dose. J Appl Clin Med Phys 2018;19:718-23.

[5] Burton CS, Szczykutowicz TP. Evaluation of AAPM Reports 204 and 220: Estimation of effective diameter, water-equivalent diameter, and ellipticity ratios for chest, abdomen, pelvis, and head CT scans. J Appl Clin Med Phys 2018;19:228-38.

[6] Anam C, Haryanto F, Widita R, Arif I, Dougherty G, McLean D. The impact of patient table on size-specific dose estimate (SSDE). Australas Phys Eng Sci Med 2017;40:153-8.

[7] Nania A, Weir A, Weir N, Ritchie G, Rofe C, Van Beek E. CTPA protocol optimisation audit: 
challenges of dose reduction with maintained image quality. Clin Radiol 2018;73:320.e1-320.e8.

[8] Laqmani A, Kurfürst M, Butscheidt S, Sehner S, Schmidt-Holtz J, Behzadi C, et al. CT pulmonary angiography at reduced radiation exposure and contrast material volume using iterative model reconstruction and iDose4 technique in comparison to FBP. PLoS One 2016;11:1-15.

[9] Sabel BO, Buric K, Karara N, Thierfelder KM, Dinkel J, Sommer WH, et al. High-pitch CT pulmonary angiography in third generation dual-source CT: Image quality in an unselected patient population. PLoS One 2016;11:1-11.

[10] Karim MKA, Hashim S, Bakar KA, Bradley DA, Ang WC, Bahrudin NA, et al. Estimation of radiation cancer risk in CT-KUB. Radiat Phys Chem 2017;137:130-4.

[11] Brenner DJ, Hall EJ. Computed tomography--an increasing source of radiation exposure. N Engl J Med 2007;357:2277-84.

[12] Laqmani A, Regier M, Veldhoen S, Backhaus A, Wassenberg F, Sehner S, et al. Improved image quality and low radiation dose with hybrid iterative reconstruction with $80 \mathrm{kV}$ CT pulmonary angiography. Eur J Radiol 2014;83:1962-9.

[13] Kim EY, Kim TJ, Goo JM, Kim HY, Lee JW, Lee S, et al. Size-specific dose estimation in the Korean lung cancer screening project: Does a 32-cm diameter phantom represent a standard-sized patient in korean population? Korean J Radiol 2018;19:1179-86.

[14] Samei E, Richard S. Assessment of the dose reduction potential of a model-based iterative reconstruction algorithm using a task-based performance metrology. Med Phys 2015.

[15] Joemai RMS, Veldkamp WJH, Kroft LJM, Hernandez-Giron I, Geleijns J. Adaptive iterative dose reduction $3 \mathrm{~d}$ versus filtered back projection in CT: Evaluation of image quality. Am J Roentgenol 2013;201:1291-7.

[16] Sookpeng S, Martin CJ, Gentle DJ. Comparison of different phantom designs for CT scanner automatic tube current modulation system tests. J Radiol Prot 2013;33:735-61.

[17] Kalra MKM, Maher MM, Toth TTL, Schmidt B, Westerman BL, Morgan HT, et al. Techniques and applications of automatic tube current modulation for CT. Radiology 2004;233:649-57.

[18] Smith-Bindman R, Lipson J, Marcus R, Kim K-P, Mahesh M, Gould R, et al. Radiation dose associated with common computed tomography examinations and the associated lifetime attributable risk of cancer. Arch Intern Med 2009;169:2078-86.

[19] Isa IN., Rahmat SMS, Dom SM, Kayun Z, Karim MK. The effects of mis-centering on radiation dose during CT head examination: A phantom study. J Xray Sci Technol 2019;Preprint:1-9.

[20] Ang WC, Hashim S, Khalis M, Karim A, Ashiqin N, Salehhon N. Adaptive iterative dose reduction ( AIDR ) 3D in low dose CT abdomen-pelvic : Effects on image quality and radiation exposure. J Phys Conf Ser 2017;012006:1-2.

[21] Alhailiy AB, Ekpo EU, Ryan EA, Kench PL, Brennan PC, McEntee MF. Diagnostic Reference Levels for Cardiac Ct Angiography in Australia. Radiat Prot Dosimetry 2018;1:1-7.

[22] Sookpeng S, Martin CJ, Gentle DJ. Investigation of the influence of image reconstruction filter and scan parameters on operation of automatic tube current modulation systems for different CT scanners. Radiat Prot Dosimetry 2015;163:521-30.

[23] Dane B, Patel H, O’Donnell T, Girvin F, Brusca-Augello G, Alpert JB, et al. Image Quality on Dual-energy CTPA Virtual Monoenergetic Images: Quantitative and Qualitative Assessment. Acad Radiol 2018;25:1075-86.

[24] Kalender WA. Dose in x-ray computed tomography. Phys Med Biol 2014;59:R129-50.

[25] Brix G, Nagel HD, Stamm G, Veit R, Lechel U, Griebel J, et al. Radiation exposure in multi-slice versus single-slice spiral CT: results of a nationwide survey. Eur Radiol 2003;13:1979-91.

[26] Karim MKA, Hashim S, Bradley DA, Bakar KA, Haron MR, Kayun Z. Radiation doses from computed tomography practice in Johor Bahru, Malaysia. Radiat Phys Chem 2016;121:69-74.

[27] Bashier EH, Suliman II. Multi-slice CT examinations of adult patients at Sudanese hospitals: radiation exposure based on size-specific dose estimates (SSDE). Radiol Medica 2018;123:424-

[28] Karim MKA, Rahim NA, Matsubara K, Hashim S, Mhareb MHA, Musa Y. The effectiveness of 
bismuth breast shielding with protocol optimization in CT scans of the thorax. J Xray Sci Technol 2018;27:1-9.

[29] Saltybaeva N, Martini K, Frauenfelder T, Alkadhi H. Organ Dose and Attributable Cancer Risk in Lung Cancer Screening with Low-Dose Computed Tomography. PLoS One 2016;11:1-11.

[30] Lahham A, ALMasri H, Kameel S. Estimation of female radiation doses and breast cancer risk from chest CT examinations. Radiat Prot Dosimetry 2018;179:303-9.

[31] Karim MKA, Hashim S, Sabarudin A, Bradley DA, Bahruddin NA. Evaluating organ dose and radiation risk of routine CT examinations in Johor Malaysia. Sains Malaysiana 2016;45:567-73. 


\section{Table}

Table 1

Data on baseline characteristic based on gender

\begin{tabular}{cccc}
\hline \multirow{2}{*}{$\begin{array}{c}\text { Baseline } \\
\text { Characteristic }\end{array}$} & Male & Female & TOTAL \\
\cline { 2 - 4 } & $49.26 \pm 14.57$ & $48.60 \pm 19.12$ & $48.88 \pm 17.28$ \\
Age* & $21.68 \pm 3.68$ & $21.88 \pm 2.71$ & $21.80 \pm 3.14$ \\
AP $(\mathrm{cm})^{*}$ & $33.46 \pm 4.17$ & $32.85 \pm 3.53$ & $33.10 \pm 3.80$ \\
LAT $(\mathrm{cm})^{*}$ & & & \\
Effective & $26.89 \pm 3.71$ & $26.76 \pm 2.64$ & $26.82 \pm 3.12$ \\
Diameter $(\mathrm{cm})^{*}$ & & & \\
\hline
\end{tabular}

$*($ mean $\pm \mathrm{SD})$ 
Table 2

Data on scanning acquisition in CTPA examination

\begin{tabular}{lc}
\hline Scanning Parameter & Values \\
\hline Tube Voltage (Kv) & 100 and $120 \mathrm{kV}$ \\
Tube Current (mAs)* & $184.50 \pm 81.87$ \\
Scan Range (mm)* & $277.44 \pm 88.03$ \\
Pitch Factor & 0.798 \\
$\begin{array}{l}\text { Beam Collimation } \\
\text { (mm) }\end{array}$ & $0.625 \times 40$ \\
Rotation Time (s) & 0.50 \\
\hline (mean \pm SD)
\end{tabular}


Table 3

Overview of the CTDI ${ }_{\mathrm{vol}}$, DLP and E value from different group of patient's effective diameter

\begin{tabular}{cccc}
\hline $\begin{array}{c}\text { Effective } \\
\text { Diameter } \\
(\mathrm{cm})\end{array}$ & $\begin{array}{c}\mathrm{CTDI}_{\mathrm{vol}} \\
(\mathrm{mGy}) *\end{array}$ & $\begin{array}{c}\text { DLP } \\
(\mathrm{mGy} . \mathrm{cm}) *\end{array}$ & $\mathrm{E}(\mathrm{mSv})^{*}$ \\
\cline { 2 - 4 } $19-25$ & $6.44 \pm 2.63$ & $239.59 \pm 97.36$ & $5.19 \pm 2.50$ \\
$25-28$ & $9.86 \pm 6.46$ & $351.85 \pm 231.85$ & $7.47 \pm 4.11$ \\
$28-38$ & $17.42 \pm 6.90$ & $631.46 \pm 274.43$ & $13.90 \pm 5.66$ \\
TOTAL & $11.06 \pm 7.17$ & $400.38 \pm 259.10$ & $8.68 \pm 5.47$ \\
\hline *(mean \pm SD $)$ & & &
\end{tabular}


Table 4

A comparison of SSDE value obtained from AAPM and CT-Expo with its ratio to CTDI ${ }_{\text {vol }}$

\begin{tabular}{|c|c|c|c|c|}
\hline \multirow{2}{*}{$\begin{array}{l}\text { Effective } \\
\text { diameter }(\mathrm{cm})\end{array}$} & \multicolumn{4}{|c|}{ Dose descriptors } \\
\hline & ${ }^{\mathrm{a} S S D E}$ (mGy) & ${ }^{\mathrm{b}} \mathrm{SSDE}(\mathrm{mGy})^{*}$ & $\begin{array}{c}\text { Ratio } \\
{ }^{\mathrm{a} S S D E} / \mathrm{CTDI} \\
\mathrm{vol}\end{array}$ & $\begin{array}{c}\text { Ratio } \\
{ }^{\text {bSSDE} / C T D I}{ }_{\mathrm{vo}}\end{array}$ \\
\hline $19-25$ & $9.93 \pm 3.89$ & $9.01 \pm 3.78$ & 1.54 & 1.30 \\
\hline $25-28$ & $13.70 \pm 9.04$ & $13.41 \pm 7.74$ & 1.42 & 1.34 \\
\hline $28-38$ & $22.29 \pm 7.35$ & $23.98 \pm 9.63$ & 1.28 & 1.31 \\
\hline \multirow[t]{2}{*}{ TOTAL } & $14.62 \pm 8.41$ & $15.37 \pm 9.67$ & 1.41 & 1.32 \\
\hline & $\begin{array}{l}{ }^{\mathrm{a}} \mathrm{SSDE}=\text { the } \mathrm{v} \\
{ }^{\mathrm{b}} \mathrm{SSDE}=\text { the } \mathrm{v} \\
*(\text { mean } \pm \mathrm{SD})\end{array}$ & $\begin{array}{l}\text { obtained from } \mathrm{A} \\
\text { obtained from } \mathrm{C}\end{array}$ & $\begin{array}{l}204 \text { report } \\
\text { oo calculator }\end{array}$ & \\
\hline
\end{tabular}


Table 5

Radiation dose comparison between this study with other studies

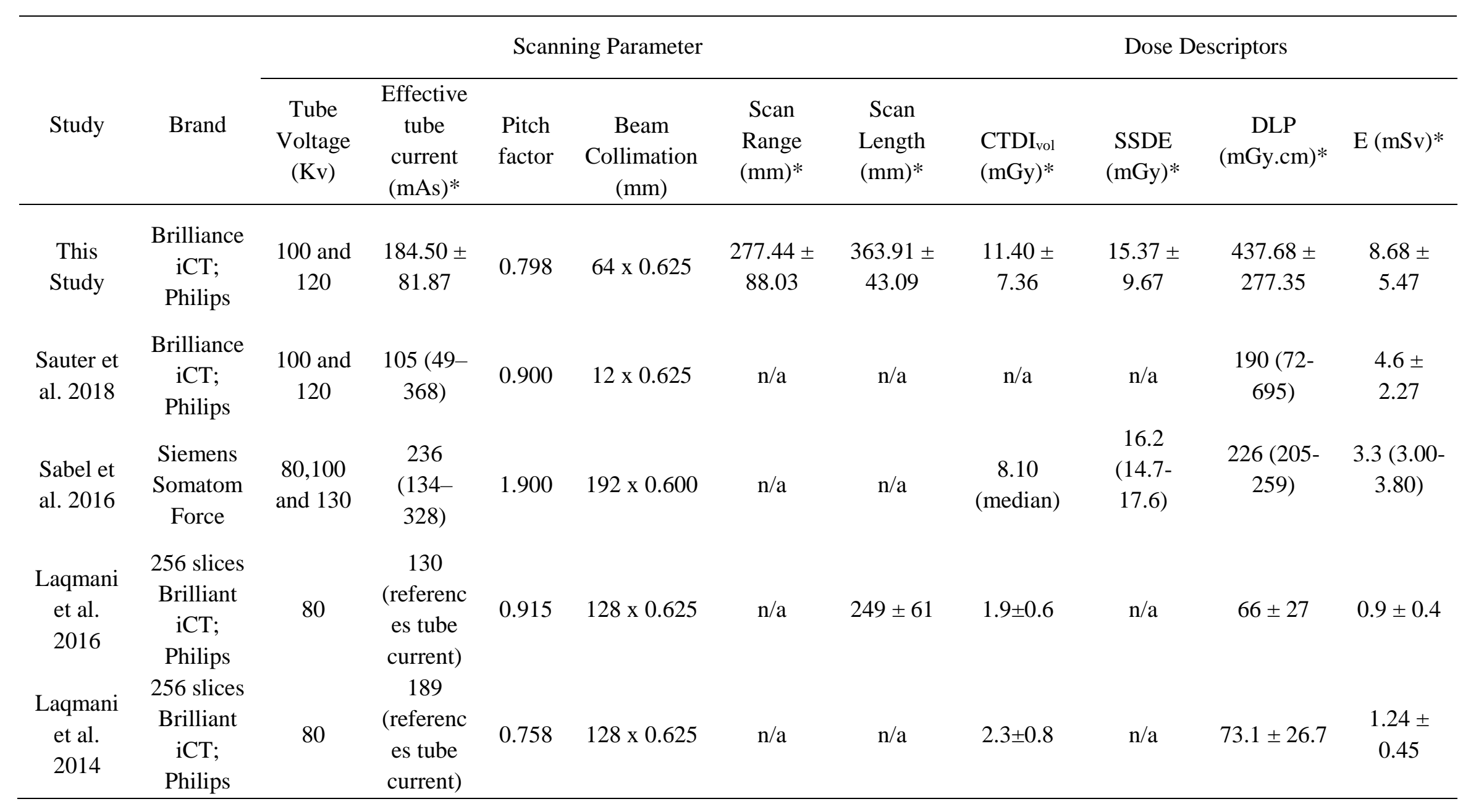


Table 6

Comparison of the mean value of organ equivalent dose based on patient's effective diameter

\begin{tabular}{cccc}
\hline \multirow{2}{*}{$\begin{array}{c}\text { Effective } \\
\text { Diameter } \\
(\mathrm{cm})\end{array}$} & Breast & Lung & Lrgan equivalent dose $(\mathrm{mSv})^{*}$ \\
\cline { 2 - 4 } $19-25$ & $10.94 \pm 4.62$ & $10.62 \pm 4.12$ & $9.15 \pm 4.23$ \\
$25-28$ & $15.48 \pm 7.93$ & $15.55 \pm 8.39$ & $13.48 \pm 6.99$ \\
$28-38$ & $23.81 \pm 12.17$ & $27.39 \pm 11.87$ & $23.19 \pm 11.76$ \\
p - value & 0.0001 & 0.0001 & 0.0001 \\
TOTAL & $17.05 \pm 10.40$ & $17.55 \pm 10.86$ & $15.04 \pm 9.75$ \\
\hline
\end{tabular}

*(mean $\pm \mathrm{SD})$ 
Table 7

Estimation of cancer probability risk according to the CTPA examination in million procedures

\begin{tabular}{|c|c|c|c|c|}
\hline \multirow{3}{*}{$\begin{array}{c}\text { Effective } \\
\text { Diameter } \\
\quad(\mathrm{cm})\end{array}$} & & \multicolumn{3}{|c|}{ Cancer Probability Risk (in million procedures) $*$} \\
\hline & & Breast & Lung & Liver \\
\hline & $\begin{array}{c}\text { Nominal } \\
\text { Risk Factor } \\
\left(r_{T}\right) 10^{-4} \mathrm{~Sv}^{-1} \\
\end{array}$ & 112.10 & 114.20 & 30.30 \\
\hline $19-25$ & & $118.67 \pm 54.45$ & $114.14 \pm 47.03$ & $31.55 \pm 12.88$ \\
\hline $25-28$ & & $174.38 \pm 94.26$ & $167.06 \pm 91.60$ & $46.59 \pm 25.63$ \\
\hline $28-38$ & & $278.63 \pm 118.27$ & $305.67 \pm 120.17$ & $84.39 \pm 33.77$ \\
\hline $\mathrm{p}$ - value & & 0.0001 & 0.0001 & 0.0001 \\
\hline TOTAL & & $194.00 \pm 113.84$ & $191.91 \pm 118.36$ & $53.19 \pm 32.90$ \\
\hline
\end{tabular}

*(mean $\pm \mathrm{SD})$ 
Table 8

Comparison based on different gender according to the CTPA examination

\begin{tabular}{ccccc}
\hline \multirow{2}{*}{ Variable } & & \multicolumn{2}{c}{ Gender } & P - value \\
\cline { 3 - 5 } & & Male & Female & 0.04 \\
\hline E $(\mathrm{mSv})^{*}$ & & $7.47 \pm 4.11$ & $9.53 \pm 5.68$ & $\mathrm{n} / \mathrm{a}$ \\
& Breast & $\mathrm{n} / \mathrm{a}$ & $17.05 \pm 10.40$ & 0.31 \\
Organ Dose $(\mathrm{mSv})^{*}$ & Lung & $15.55 \pm 8.39$ & $17.63 \pm 10.41$ & 0.84 \\
& Liver & $13.48 \pm 6.99$ & $14.58 \pm 9.28$ & $\mathrm{n} / \mathrm{a}$ \\
Cancer Probability & Brest & $\mathrm{n} / \mathrm{a}$ & $194.00 \pm 113.84$ & 0.16 \\
Risk (per one million & Lung & $185.54 \pm 124.36$ & $196.53 \pm 114.70$ & 0.16 \\
\hline
\end{tabular}

*(mean $\pm \mathrm{SD})$ 
Figure
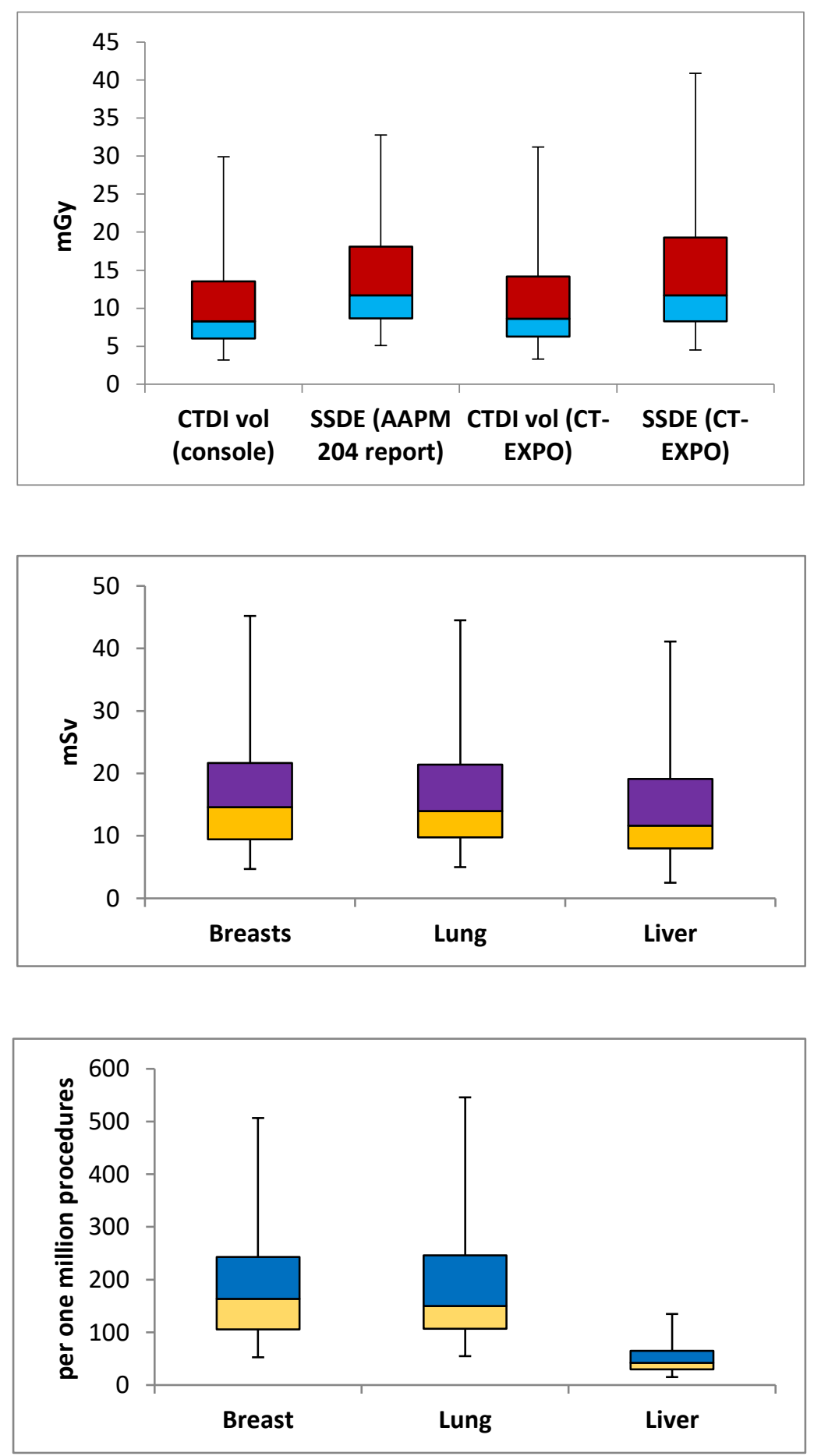

Fig. 3. The distribution of radiation dose and cancer probability risk value in CTPA examination: (a) CTDI $_{\text {vol }}$ vs SSDE (b) Organ Dose (c) Effective Cancer Risk. 